\title{
The Mammalian Circadian Clock in the Suprachiasmatic Nuclei Is Reset in vitro by CAMP
}

\author{
Rebecca A. Prosser and Martha U. Gillette \\ The Neural and Behavioral Biology Program and Department of Physiology and Biophysics, University of Illinois at \\ Urbana-Champaign, Urbana, Illinois 61801
}

\begin{abstract}
A circadian clock located in the suprachiasmatic nuclei (SCN) of the hypothalamus controls the daily behavioral and physiological rhythms of mammals. While the mammalian circadian system has been the focus of research for many years, very little work has been directed at understanding its underlying biochemical mechanisms. In these experiments we used the hypothalamic brain slice technique to investigate these mechanisms, focusing specifically on the intrinsic resetting properties of the circadian clock of the rat. We monitored a primary expression of the clock or pacemaker, the circadian rhythm of electrical activity of SCN neurons. This rhythm continues the oscillatory pattern seen in vivo for up to $60 \mathrm{hr}$ in vitro, with an activity peak near midday that shows very little variation among SCN from different rats. The stability of the rhythm in vitro enabled us to use the time of peak activity to monitor the phase of the underlying pacemaker. Bath application of membrane-soluble cAMP analogs in $1 \mathrm{hr}$ pulses induced robust advances in the phase of the rhythm that remained stable for 2 cycles. This effect depended on the phase of the pacemaker at the time of treatment: the peak was maximally advanced (4-6 hr) by treatments during the middle of the subjective day (projected from the donor's cycle); treatments during most of the subjective night and early subjective day induced no phase changes. Half-maximal phase resetting was induced at $1 \times$ $10^{-10} \mathrm{M}$ concentrations of active cAMP analog. The pacemaker showed similar temporal sensitivity to treatments that increase endogenous CAMP (forskolin, RO 20-1724, IBMX), but was unaffected by a non-cyclic 5'-AMP analog. Together, these results demonstrate that (1) the SCN contain a selfsustaining pacemaker that survives removal from the animal and can be reset in vitro, and (2) this pacemaker is rapidly reset by daytime increases in CAMP. These data show that CAMP pathways have access to the entraining mechanism(s) of the clock and are potentially elements of the clock itself. In addition, the finding that the pacemaker can be reset in vitro demonstrates the usefulness of the brain slice preparation to study biochemical substrates of the mammalian circadian clock.
\end{abstract}

\footnotetext{
Received May 4, 1988; revised July 15, 1988; accepted July 22, 1988.

We wish to thank Drs. Rhanor Gillette and Patricia DeCoursey for their helpful discussions. This work was supported by PHS Grant NS 22155 to M.U.G.

Correspondence should be addressed to Martha U. Gillette, Department of Physiology and Biophysics, University of Illinois at Urbana-Champaign, 524 Burrill Hall, 407 S. Goodwin, Utrbana, IL 61801

Copyright (C) 1989 Society for Neuroscience $0270-6474 / 89 / 031073-09 \$ 02.00 / 0$
}

Mammals, like many other eukaryotic organisms, exhibit daily rhythms in their behavior and physiology that are controlled by an endogenous biological clock that keeps near-24-hr time. The environmental lighting cycle synchronizes these daily rhythms with the solar cycle by regulating the phase of this internal clock. Although this system has been studied for many years, the cellular mechanisms underlying this endogenous clock, or pacemaker, are largely unknown.

The primary mammalian circadian pacemaker is located at the base of the brain in the suprachiasmatic nuclei (SCN) (Moore and Eichler, 1972; Stephan and Zucker, 1972). Because of its inaccessibility in vivo, the endogenous properties of the pacemaker have been most effectively studied by maintaining it in vitro. In such a preparation the $\mathrm{SCN}$ are isolated from sensory as well as CNS afferents and thus behave according to properties contained only in the cultured tissue. Brain slice culture of SCN for conventional periods (up to $8 \mathrm{hr}$ ) has revealed that neuronal activity and glucose utilization of $\mathrm{SCN}$ in vitro are high in the day and low at night (Groos and Hendricks, 1982; Shibata et al., 1982; Shibata and Moore, 1988). Further, SCN neuronal sensitivities to a variety of neurotransmitters have been directly demonstrated in vitro (Nishino and Koizumi, 1977; Groos et al., 1983).

In order to examine circadian pacemaking properties, however, the tissue must be maintained in vitro for at least $24 \mathrm{hr}$. Studies that accomplished this revealed that the SCN sustain circadian rhythms of neuronal firing rate (Green and Gillette, 1982 ) and secretion of the neuropeptide vasopressin (Earnest and Sladek, 1986, 1987; Gillette and Reppert, 1987). The in vitro rhythm in firing rate of the population of single SCN neurons is similar to the rhythm of multiunit activity recorded in vivo (Inouye and Kawamura, 1979), with peak activity near midday of the donor's entraining light period (the subjective day of the slice) and a trough during the donor's dark period (subjective night). For rats from our inbred colony, the time of this peak during the first $24 \mathrm{hr}$ in vitro was found to be stable among SCN from different donors (Gillette, 1985); longer periods had not been explored.

The stability of this rhythm allowed us to observe that stimuli present during brain slice preparation changed the time of the next electrical activity peak in vitro (Gillette, 1986). The timeof-peak was altered by tissue preparation only during subjective night, the period when the animal is sensitive to the resetting effects of light. However, to demonstrate that in vitro shifts in phase reflect permanent resetting of the pacemaker mechanism, it remained to observe a sustained shift in subsequent circadian cycles. 
Thus, in the present study we first sought to determine whether the circadian rhythm in SCN neuronal firing rate was maintained for multiple cycles in vitro. This would further establish that the pacemaker is endogenous to the isolated tissue and would assess the stability of the endogenous rhythm over several days outside of the animal. We could then justify using the time of peak neuronal activity as a measure of the phase of the unperturbed pacemaker over multiple cycles.

$\mathrm{Next}$, in order to investigate the involvement of identifiable biochemical pathways in the pacemaker, we tested the ability of specific biochemical agents to induce phase shifts of this endogenous circadian rhythm in vitro. By observing the response of the system over several cycles, we hoped to establish whether changes were stable shifts, indicating true resetting of the pacemaker mechanism.

In preliminary studies we reported that the $\mathrm{SCN}$ in vitro are sensitive during the subjective day but not subjective night to analogs that mimic cAMP (Prosser and Gillette, 1986; Gillette and Prosser, 1988). We report here that in vitro treatments that selectively stimulate cAMP-dependent pathways induce rapid, stable phase shifts of the isolated mammalian pacemaker. We establish the relationship between the circadian time of $1 \mathrm{hr}$ pulses and the response of the oscillation in neuronal activity, and we report a dose-response relationship for one cAMP analog.

\section{Materials and Methods}

Brain slice culture. Brain slices were prepared during the donor's day from 2- to 5-month-old Long-Evans rats born in our inbred colony and reared in a 12:12 light-dark (LD) cycle. Animals were housed and handled with care, in full accordance with NIH and institutional guidelines. The procedures used to prepare, maintain, and record from the brain slices have been described previously (Gillette, 1986; Gillette and Reppert, 1987). Briefly, $500 \mu \mathrm{m}$ coronal slices of the hypothalamus containing the paired SCN, optic chiasm, third ventricle, and surrounding tissue were prepared during lights-on, and placed in the center of a Hatton-style brain slice chamber (Hatton et al., 1980). The slices were continuously perifused at $35 \mathrm{ml} / \mathrm{hr}$ with Earle's Minimal Salt Solution (GIBCO) supplemented with $24.6 \mathrm{~mm}$ glucose and $26.2 \mathrm{~mm}$ sodium bicarbonate. The medium was adjusted to $\mathrm{pH} 7.2$, warmed to $37^{\circ} \mathrm{C}$, and saturated with $95 \% \mathrm{O}_{2} / 5 \% \mathrm{CO}_{2}$. The chamber was continuously illuminated with a fiberoptic light (American Optical). In experiments in which slices werc maintaincd for morc than $36 \mathrm{hr}, 0.5 \%$ Gentamicin was added to the perifusion medium, and the medium was sterilized by filtration.

Neurophysiological recording. Extracellular recordings from single cells were made using glass microelectrodes filled with $5 \mathrm{M} \mathrm{NaCl}$. An electrode was lowered into the $\mathrm{SCN}$ using an hydraulic microdrive until the signal from a single cell was encountered. Electrical signals achieving or exceeding twice the level of the background noise were isolated using a window discriminator, observed for stability over at least $2 \mathrm{~min}$, and sent to a computer for counting. The firing rate of each cell was monitored over sequential $120 \mathrm{sec}$ periods, using $10 \mathrm{sec}$ bins. The electrode was then repositioned until another cell was encountered at random.

Phase-shifting treatments. Slices were allowed to equilibrate for at least $1 \mathrm{hr}$ in the recording chamber before chemical treatment. The RO 20-1724 and forskolin were kindly donated by Dr. Rhanor Gillette. 8-benzylamino-cyclic adenosine monophosphate (BA-cAMP) and 8-bromocyclic adenosine monophosphate (Br-cAMP), both membranepermeable cAMP analogs that are potent stimulators of cAMP-dependent protein kinases and resist metabolic degradation (Meyer and Miller, 1974); 8-bromo-adenosine monophosphate (Br-5'-AMP); and isobutylmethylxanthine (IBMX) were obtained from Sigma.

Less than 20 min before treatment, the test substance was dissolved in normal perifusion medium, the $\mathrm{pH}$ was adjusted to 7.2 , and the medium was warmed and bubbled with $95 \% \mathrm{O}_{2} / 5 \% \mathrm{CO}_{2}$. At the appropriate time, perifusion was stopped, and the normal medium was replaced in less than 2 min with medium containing the substance to be tested. After $1 \mathrm{hr}$, the treatment medium was exchanged completely
Table 1. Treatment of SCN in vitro with BA-cAMP at CT 7-8 induces a significant shift in subsequent cycles of neuronal firing rate compared with untreated SCN

Mean time (CT) of peak electrical activity in vitro

\begin{tabular}{llll} 
Group & Day 1 & Day 2 & Day 3 \\
\hline Untreated & $7.1 \pm 0.1$ & $6.9 \pm 0.2$ & $6.6 \pm 0.4$ \\
& $(n=3)$ & $(n=8)$ & $(n=3)$ \\
BA-cAMP & - & $2.4 \pm 0.2^{b}$ & $\begin{array}{l}2.2 \pm 0.4^{a} \\
(n=3)\end{array}$ \\
\hline
\end{tabular}

"p<0.01 compared with untreated day 3 .

$p<0.001$ compared with untreated day 2 .

for normal medium, and perifusion was resumed. Electrical activity was then monitored during the subsequent 1 or 2 circadian cycles to determine the timc of the peak(s) in neuronal firing.

Data analysis. As a preliminary analysis, the firing rates of individual SCN neurons recorded during each experiment were grouped into $2 \mathrm{hr}$ running averages using $1 \mathrm{hr}$ lags. The time-of-peak was then determined by visual inspection of a graph of these values for the symmetrically highest point. Subsequently, the data were analyzed in more detail by plotting the $2 \mathrm{hr}$ averages using $15 \mathrm{~min}$ lags. The time-of-peak was again determined visually by one of the experimenters without prior knowledge of the experimental conditions to insure against bias. The reliability of this method in estimating the phase of the underlying pacemaker was reinforced by the fact that the time-of-peak estimates produced by these 2 analyses differed, on average, by less than 15 min per experiment over more than 80 experiments, with a maximum discrepancy of $1 \mathrm{hr}$

Phase shifts were determined by comparing the time of peak electrical activity in treated slices with that of untreated slices. Differences between groups were evaluated using Student's $t$ test.

\section{Results}

\section{Circadian properties of SCN slices in long-term culture}

In order to determine whether SCN maintained in vitro are able to generate multiple, stable oscillations in electrical activity, we monitored the pattern of electrical activity during the subjective day for up to 3 circadian cycles. In agreement with our earlier work (Gillette, 1985, 1986), the mean time-of-peak on day 1 in culture for slices prepared before the peak on the first day was at circadian time $7.1 \pm 0.1$ (CT 7.1, where CT 0 corresponds to lights-on in the donor's LD cycle and continues for $24 \mathrm{hr}$ before starting over).

When SCN were maintained in vitro for up to $60 \mathrm{hr}$, they continued the circadian pattern of neuronal activity exhibited during the first day in vitro: activity continues to rise and fall with a peak near midday. Analysis of the time-of-peak both within and across long-term experiments (Fig. 1) revealed that peak time, and thus the phase of the pacemaker, remained relatively stable over cycles 2 and 3 . In most experiments, little damping of the amplitude of the rhythm was observed during this period (Fig. 1, $A, B$ ). The damping that appears in the cumulative data for day 3 (Fig. $1 C$ ) may be due to variation in the health of the tissue by day 3 and slightly greater variation in peak time on day $3( \pm 0.4 \mathrm{hr}$, Table 1$)$.

In our culture conditions the time-of-peak was not significantly different between consecutive days in vitro. Comparing the mean time-of-peak on day 3 with that on day 1 suggests that the long-term trend is toward a daily period of slightly less than $24 \mathrm{hr}$ under these culture conditions (Table 1). The ability of SCN to maintain a stable, robust circadian rhythm of electrical activity for at least $60 \mathrm{hr}$ in vitro allows us to use this preparation to investigate resetting properties of the pacemaker. 


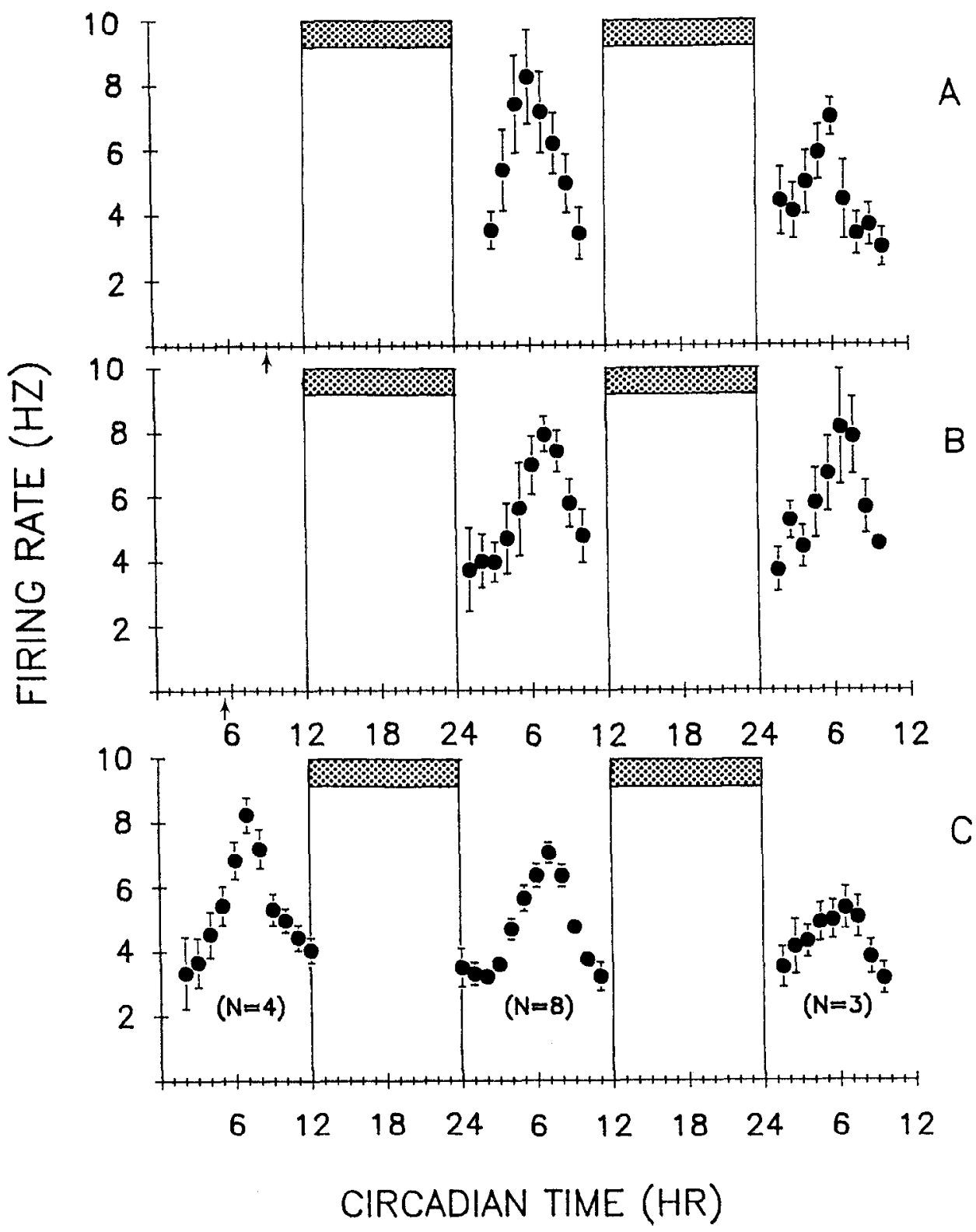

Figure 1. Neuronal electrical activity rhythm continues for multiple cycles in vitro. $A$ and $B$, Plotted are the 2 hr means \pm SEM of all single units recorded on days 2 and 3 in single experiments. The running averages shown (using $1 \mathrm{hr}$ lags) were chosen on the basis that they illustrate the most symmetrical pattern of activity around the peak. In the experiment shown in $A$, electrical activity peaked at CT 6 on both days 2 and 3 . In $B$, the rhythm peaked at CT 7 on day 2 and CT 6.5 on day 3. $C$, Summed data from all control experiments, showing the stability of the electrical activity rhythm during the first $3 \mathrm{~d}$ in vitro. $N=$ the number of experiments contributing to the data for each day; horizontal bars indicate subjective night for the SCN, the time of lights-off in the donor colony; and arrows indicate the time of slice preparation.

\section{Effects of $1 \mathrm{hr}$ pulses of $B A-c A M P$}

The sensitivity of the isolated pacemaker to resetting of its phase in vitro was examined by applying discrete $1 \mathrm{hr}$ pulses of a membrane-permeable cAMP analog to $\mathrm{SCN}$ in brain slices. The rhythm of firing rate was then examined as in control cultures. When $5 \times 10^{-4}$ M BA-cAMP was applied early in the subjective day, we found that it produced transient effects on the electrical activity rhythm without affecting the pacemaker. This can be seen in experiments in which BA-cAMP was applied at CT 1.52.5 (Fig. 2): the pattern of electrical activity directly following treatment was variable. Acute responses to cAMP iontophoretically applied to SCN neurons have been reported previously (Liou et al., 1986). However, the electrical activity during the subsequent cycle, more than $24 \mathrm{hr}$ after treatment, showed no alteration in phase. For this reason the phase of the pacemaker could not be rcliably assessed during the hours directly following treatment.
When the SCN were exposed to a $1 \mathrm{hr}$ pulse of BA-cAMP later in the subjective day, the result was strikingly different (Fig. $3 A$ ). One hour exposure of the SCN to BA-cAMP at CT $7-8$ on day 1 in vitro altered the pattern of firing rate in the next cycle (day 2 ) so that peak activity no longer occurred near CT 7; rather, the peak occurred at CT 2 . In 5 experiments examining the effect of BA-cAMP applied at CT 7-8, the mean time-ofpeak was $2.4 \pm 0.2$ (Table 1). Compared with the time-of-peak on day 2 of control experiments, this represents a mean advance of the phase by $4.5 \mathrm{hr}$. In contrast, when SCN experienced an identical treatment $12 \mathrm{hr}$ later, at CT 19-20, the subsequent peak in electrical activity occurred at CT 6.8 (Fig. $3 B$ ), the same time as seen in untreated SCN. Thus, near the middle of the donor's night, the pacemaker is again insensitive to cAMP stimulation.

To determine whether these phase advances were stable, we monitored the electrical activity rhythm for 2 cycles following treatment. Results of 3 such experiments appear in Figure 4. In 


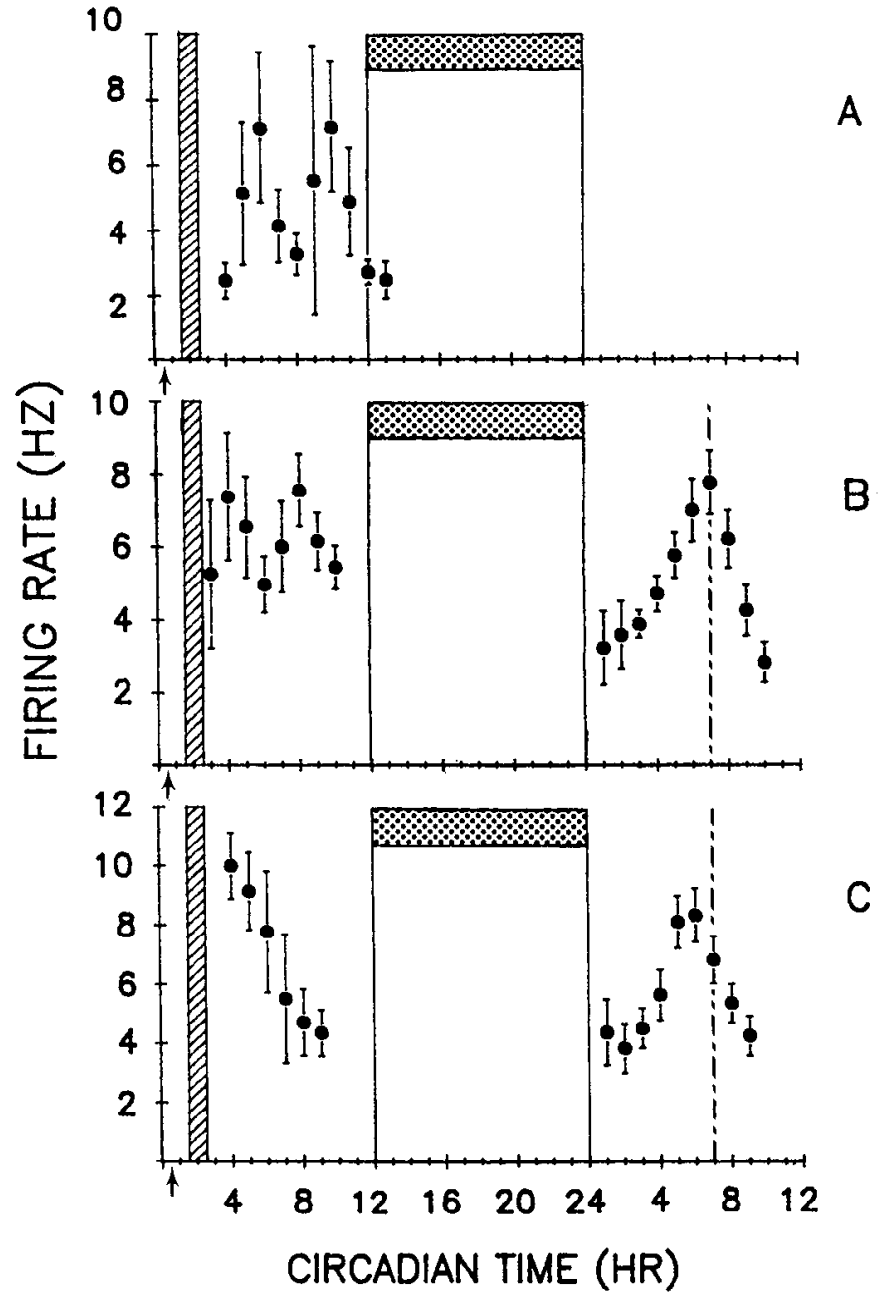

Figure 2. Transient perturbations of SCN electrical activity immediately following $5 \times 10^{-4} \mathrm{M}$ BA-cAMP treatment are distinct from longterm effects on the circadian pacemaker. $A-C$, Electrical activity following treatment at CT 1.5-2.5 shows variable patterns during the 10 $\mathrm{hr}$ aftcr trcatment. However, these unusual patterns of electrical activity are no longer present during the second day in vitro. Peaks in electrical activity at CT $6.75(B)$ and CT $5.75(C)$ during the second cycle in vitro indicate little or no phase advance of the underlying circadian pacemaker. Vertical bar indicates the time of treatment. Dotted line indicates the mean time of peak activity in untreated slices. See Figure 1 legend for explanation of other symbols.

the first experiment, SCN were treated between CT 7-8 on the first day in vitro. Brain slices were then left undisturbed until the third day, when the rhythm of firing rate was recorded. As seen in Figure $4 A$, the time of peak firing rate was at CT 2, which is $4.6 \mathrm{hr}$ earlier than in control cultures maintained for $3 \mathrm{~d}$. This shift is identical to that of SCN treated on day 1 at CT 7-8, where the peak was assessed on day 2 and suggests that the pacemaker has been completely reset by day 2 .

In other experiments, 2 consecutive cycles of neuronal activity were recorded following analog treatment. The results of these experiments confirm that the shift seen on day 2 was complete: the carly time-of-peak seen on day 2 was maintained, appearing $24 \mathrm{hr}$ later on day 3 in vitro, still advanced by more than $4 \mathrm{hr}$ compared with controls (Fig. 4, B, C; Table 1). Thus, these phase shifts appear to be induced and completed during the cycle of treatment so that when activity rises and peaks the next day, the new phase has been fully set.

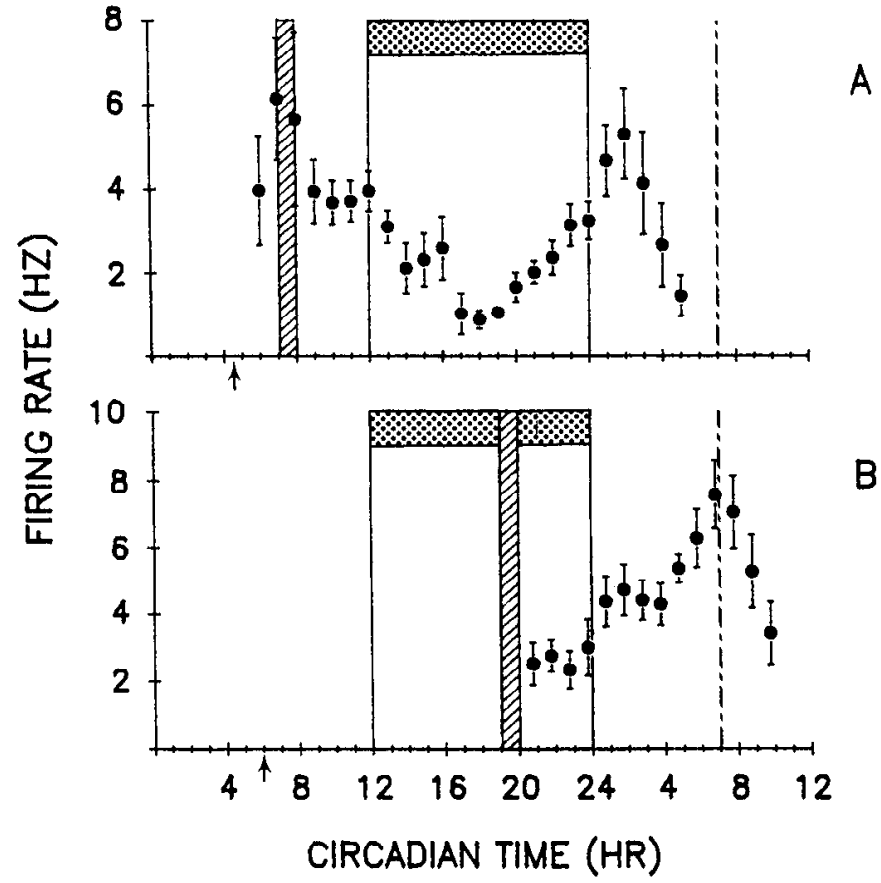

Figure 3. Phase-dependent effects of BA-cAMP treatment on the circadian electrical activity rhythm. $A$, BA-cAMP treatment at CT 7-8 shifts the electrical activity rhythm so that the time of peak activity on the second day in vitro occurs at CT 2.0 , which is $5 \mathrm{hr}$ carlicr than normal. $B$, BA-cAMP treatment at CT 19-20 does not shift the time of the subsequent peak in neuronal activity. See Figure 1 and 2 legends for explanation of symbols.

To characterize the differential sensitivity of the pacemaker to c $\Lambda \mathrm{MP}$ stimulation more fully, we examined the effect of BAcAMP applied at various points in the circadian cycle. Because the phase changes induced by BA-cAMP appear to be complete within $18 \mathrm{hr}$ after the onset of cAMP stimulation, the phase changes in this series of experiments were determined during the cycle after treatment. The resulting relationship between the circadian phase of treatment and response of the pacemaker is shown in Figure 5. The largest phase changes occurred when BA-cAMP was applied for $1 \mathrm{hr}$ between CT 3-7. These were phase advances of 5-6 hr. In contrast, when applied for $1 \mathrm{hr}$ anywhere from CT 13 through the subjective night to CT 1.5 in the subjective morning, BA-cAMP induced little or no change ( $\leq 1 \mathrm{hr}$ from controls). Simply replacing the medium with fresh medium for $1 \mathrm{hr}$ at CT 4 or 7 had no effect on the phase (mean phase advance of $0.6 \mathrm{hr} ; n=2$ ). Interestingly, we found no phase when BA-cAMP treatment delayed the pacemaker.

\section{Pacemaker sensitivity to $B A-c A M P$}

While the above manipulations were performed with $5 \times 10^{-4}$ M BA-cAMP, a concentration that stimulates a change in active state in some neurons (R. Gillette et al., 1982), this exceeds the expected intracellular concentration of cAMP (Steiner et al., 1972; Hockberger and Yamane, 1987). We therefore examined the effect of serial dilutions of the analog applied at CT 7-8. We observed that the ability of BA-cAMP to change the phase of the pacemaker is indeed dose dependent (Fig. 6). Phase advances of maximum magnitude occurred with doses as low as $5 \times 10^{-9} \mathrm{M}$. Below that, the magnitude of the phase advance declined linearly until $5 \times 10^{-12} \mathrm{M}$, when the phase was not 


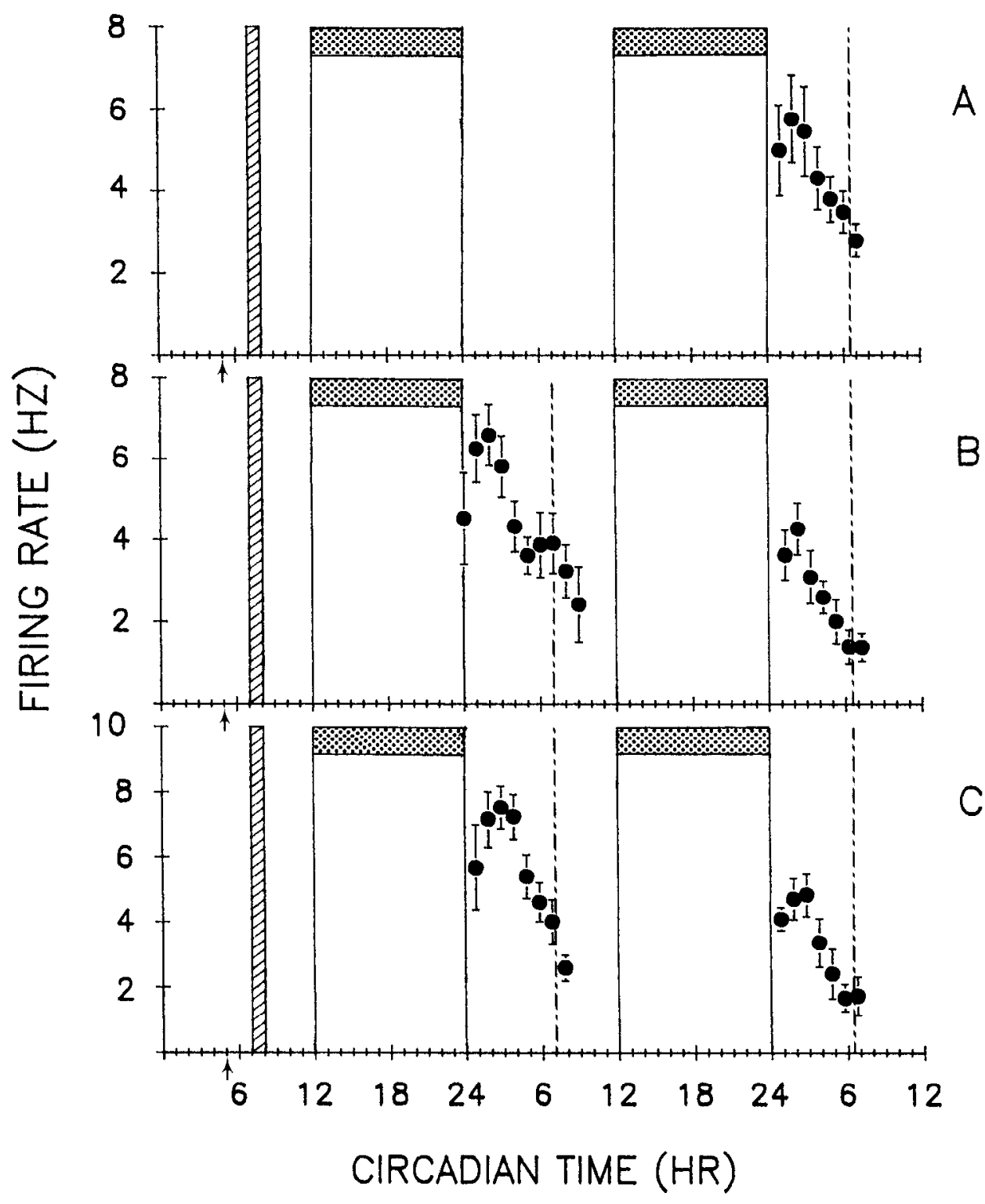

Figure 4. BA-cAMP induces stable phase advances in vitro. In separate $\mathrm{ex}$ periments, slices were treated with BAcAMP at CT 7-8 on day 1 in vitro, and electrical activity was recorded on day $3(A)$ and days 2 and $3(B$ and $C$ ) in vitro. $A$. Electrical activity peaks at $\mathrm{CT}$ 2 on day 3 in vitro. $B$, Electrical activity peaks at CT 2 on day 2 and CT 2.25 on day 3 in vitro. $C$, Electrical activity peaks at CT 2.75 on days 2 and 3 . The time of the shifted peak in activity is very consistent across experiments. These data indicate that the phase advances are complete within $24 \mathrm{hr}$ of cAMP analog treatment and represent stable shifts of the underlying circadian pacemaker. See Figure 1 and 2 legends for explanation of symbols. significantly different from controls. The point for half-maximal phase advance falls at $1 \times 10^{-10} \mathrm{M}$.

In order to address the possibility that phase delays were obscured at $5 \times 10^{-4} \mathrm{M}$, we examined the effect of $5 \times 10^{-8} \mathrm{M}$ BA-cAMP applied at CT 1.5-2.5. By extrapolation from in vivo phase-shifting experiments on rodents, the period preceding phase advances might be expected to produce delays. However, the lower concentration was ineffective at inducing a delay in a single experiment.

\section{Effects of other cAMP analogs}

If the phase-resetting effects of BA-cAMP are due specifically to its ability to mimic endogenous cAMP, then other cAMP analogs with similar properties should induce similar phase changes. Conversely, pharmacological agents that are structurally similar but do not stimulate cAMP pathways should not produce similar effects. With this in mind, we tested another cAMP analog, Br-cAMP, for its ability to affect the pacemaker. A $1 \mathrm{hr}$ pulse of $5 \times 10^{-4} \mathrm{M} \mathrm{Br}$-cAMP at CT 7-8 induced an advance in the phase of the rhythm of electrical activity of similar magnitude to that induced by BA-cAMP (Fig. $7 A$ ). In contrast, the structurally similar but non-cyclic form of this analog, Br-5'-AMP, had no effect on the phase of the rhythm (Fig. 7B, Table 2). Thus, the ability of cAMP analogs to reset the SCN pacemaker is due to their ability to mimic endogenous cAMP

\section{Effects of treatments that augment endogenous $C A M P$}

We further tested the hypothesis that daytime stimulation of $\mathrm{c} \Lambda \mathrm{MP}$ pathways mediates phase resetting in the SCN by cxamining the response to agents that raise levels of endogenous cAMP. Endogenous levels can be increased by either decreasing the rate of cAMP degradation or by increasing its rate of synthesis. Application of the general phosphodiesterase inhibitor IBMX $\left(1 \times 10^{-4} \mathrm{M}\right)$ or an inhibitor specific for the low- $k_{D}$ form of cAMP-phosphodiesterase, RO 20-1724 (5 × 10-5 M) (Chasin and Harris, 1976), at CT 7-8 each induced phase advances of $4 \mathrm{hr}$ (Fig. 8A, Table 2). Conversely, nighttime applications of RO 20-1724 caused no change in the phase of the electrical activity rhythm (Table 2 ). 


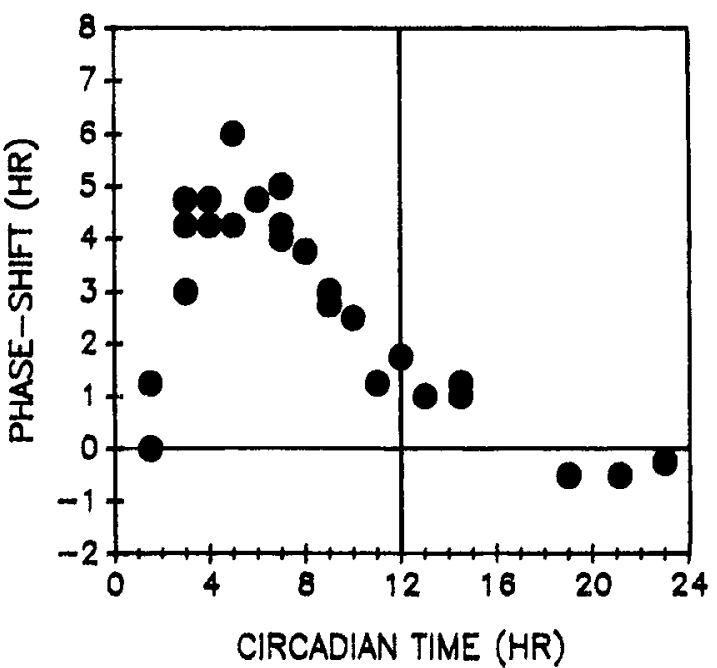

Figure 5. Relationship between the time of BA-cAMP treatment and its effect on the phase of the electrical activity rhythm. Treatments during most of the subjective day (CT 3-12) produce significant phase advances, whereas treatments during most of the subjective night produce little or no phase changes. Filled circles, phase shifts determined $1 \mathrm{~d}$ after treatment.

We also tested whether increasing endogenous cAMP levels by stimulating adenyl cyclase activity with forskolin (Seamon et al., 1981) affected the pacemaker. In vitro treatment with 1 $\times 10^{-6} \mathrm{M}$ forskolin at CT 7-8 induced a $4.3 \mathrm{hr}$ advance in the phase of the rhythm (Fig. $8 B$ ), whereas nighttime treatment did not affect it (Table 2). Thus, increasing endogenous cAMP levels has the same time-of-day-dependent effect on the SCN pacemaker as CAMP analogs that stimulate cAMP-dependent pathways in neurons.

\section{Discussion}

Our experiments were designed to begin analyzing the endogenous properties of the mammalian suprachiasmatic pacemaker. Initially, we asked whether the SCN rhythm of electrical activity could continue for several cycles in vitro and then whether the phase of the SCN rhythm could be reset outside of the organism. With respect to the first point, we found that in the perifused brain slice system, SCN retain the ability to generate multiple circadian cycles of electrical activity. The pattern of the rhythm in these conditions of culture is the same from day to day, with little damping of the peak through the third cycle. The times of peaks within individual experiments suggest that the daily period does not differ markedly from $24 \mathrm{hr}$ over the $3 \mathrm{~d}$ measured. However, the trend across experiments suggests that if measurements were carried out over more cycles, the free-running period of the rhythm in these culture conditions would be slightly less than $24 \mathrm{hr}$.

Nevertheless, both across days within a single experiment and across experiments at each day measured, the time of peak neuronal activity, and thus the phase of the oscillator, is remarkably stable. This may reflect the relative genetic homogeneity of rats (and SCN) from our colony, which has been inbred for more than 15 generations, as well as the constancy of the environment within the brain slice chamber. The stability of this rhythm allows us to use this in vitro system to approach questions regarding the mechanism underlying the SCN clock.

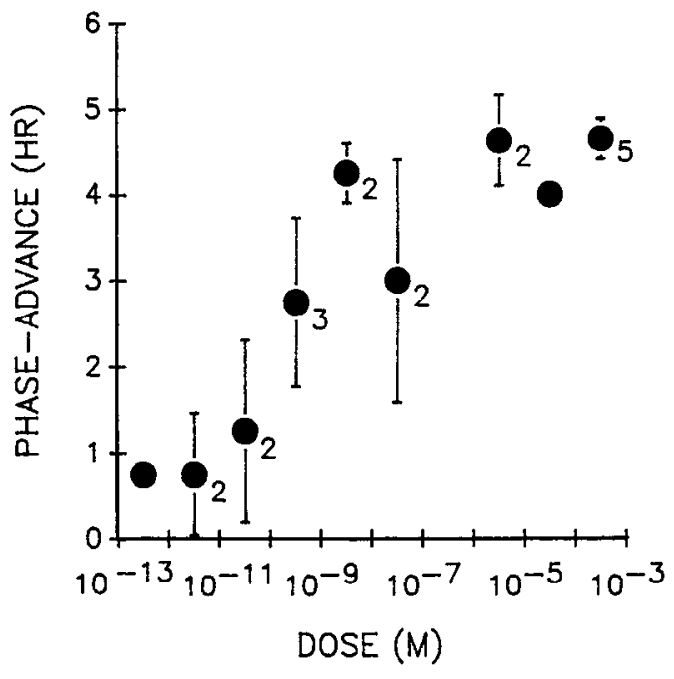

Figure 6. Dose-response curve for BA-cAMP. Plotted are mean phase advances \pm SEM of the electrical activity rhythm induced by various concentrations of BA-cAMP applied at CT 7-8. Number of replications at each concentration is noted.

To investigate the ability of the SCN pacemaker to be reset in vitro, we examined its sensitivity to a variety of treatments that affect intracellular cAMP pathways. This particular secondmessenger system was selected because of its well-established role in regulating intracellular changes of state. Our preliminary evidence suggested that the SCN pacemaker could be perturbed by cAMP analog treatment during the subjective day (Prosser and Gillette, 1986; Gillette and Prosser, 1988).

We found that the SCN pacemaker is specifically sensitive between CT 3-12 to a variety of treatments that increase intracellular cAMP levels or mimic endogenous cAMP. During this part of the subjective day, cAMP stimulation induces robust advances of up to $6 \mathrm{hr}$ in the phase of the circadian rhythm of SCN neuronal activity in vitro. These treatments have no significant effect on this rhythm when applied during the rest of the circadian cycle.

These results have meaning with respect to 2 aspects of our understanding of the SCN pacemaker: (1) They reveal characteristics of the phase-resetting process in the isolated pacemaker, and (2) the phase-dependent effects of cAMP suggest that CAMPsensitive pathways are linked with the SCN circadian pacemaker, possibly as part of the pacemaker mechanism itself or as an entraining signal. These results will be discussed in turn.

With respect to the first point, we do not know how quickly the isolated pacemaker responds to cAMP stimulation, but it appears to respond much more rapidly than predicted from in vivo work on phase shifting in rodents. Phase advances in vitro induced by cAMP analogs are complete within the first cycle: the phase of the rhythm does not continue to advance after the first cycle following treatment. We have observed stable advanced peaks as early as $18 \mathrm{hr}$ after exposure to cAMP stimulation. It seems reasonable to predict that the biochemical substrates of the pacemaker underlying the rhythm of neuronal firing rate are altered considerably earlier.

This observation contrasts with phase shifts induced in vivo, which often require several days to be completed (Pittendrigh, 1960). Characteristically, organisms in constant darkness that are exposed to a brief light pulse during the subjective night 


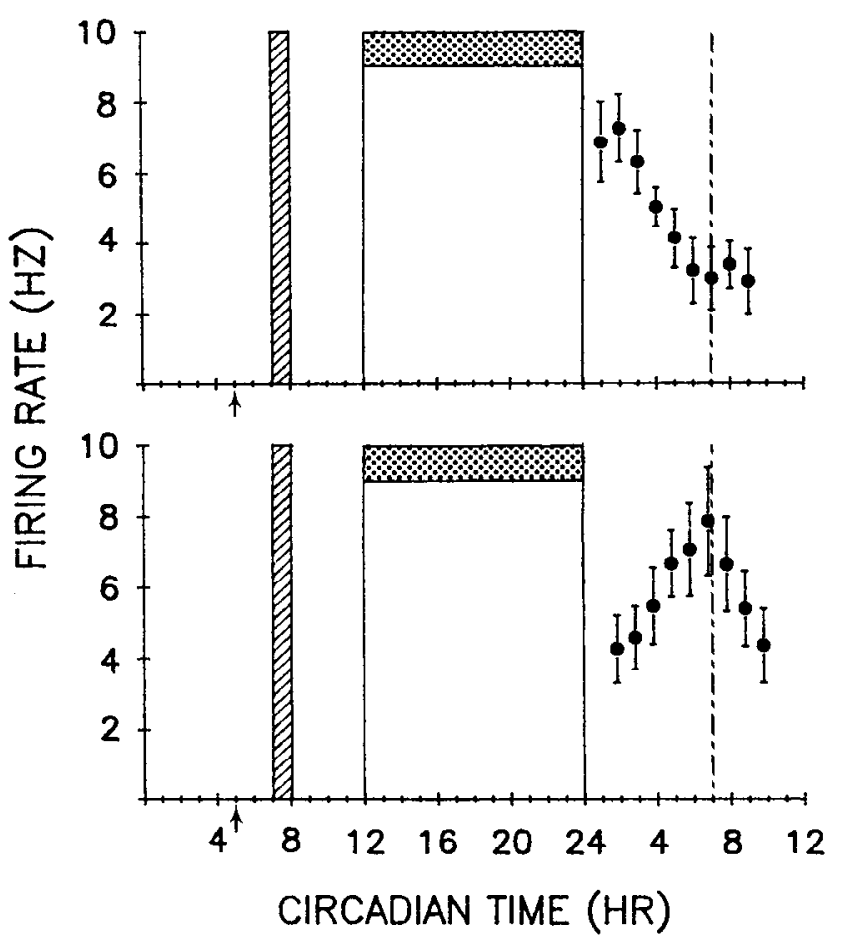

Figure 7. A, Application of another active analog, $5 \times 10^{-4} \mathrm{M} \mathrm{Br}$ CAMP, at CT 7-8 advances the electrical activity rhythm $5.0 \mathrm{hr}$; the peak 1 day after treatment is at CT $2.0 . B, \mathrm{Br}-5^{\prime}$-AMP, a structurally similar, but non-cyclic, adenosine nucleotide, does not alter the electrical activity rhythm. The electrical activity rccorded $1 \mathrm{~d}$ after treatment with $5 \times 10^{-4} \mathrm{M}$ Br-5'-AMP at CT 7-8 peaks at CT 6.75 , indicating no change in the phase of the underlying pacemaker. See Figure 1 and 2 legends for explanation of symbols.

show changes in the time of onset of behavioral activity rhythms that gradually shift over several days to a new, stable phase (DeCoursey, 1964). There is evidence, however, that even in vivo the pacemaker itself may shift in less than one cycle. Pit-

\section{Table 2. Effects of various treatments on the phase of the SCN} electrical activity rhythm in vitro

\begin{tabular}{lll} 
& \multicolumn{2}{c}{$\begin{array}{l}\text { Mean hourly phase advance induced by } \\
\text { in vitro treatments }\end{array}$} \\
\cline { 2 - 3 } Treatment & Daytime $^{\alpha}$ & Nighttime \\
\hline BA-cAMP & $4.6 \pm 0.2^{d}$ & $-0.3 \pm 0.3^{\prime \prime}$ \\
$\quad\left(5 \times 10^{-4} \mathrm{M}\right)$ & $(n=5)$ & $(n=7)$ \\
Br-cAMP & 4.3 & - \\
$\left(5 \times 10^{-5} \mathrm{M}\right)$ & $(n=2)$ & - \\
Br-5'-AMP & 0.4 & \\
$\left(1 \times 10^{-4} \mathrm{M}\right)$ & $(n=2)$ & $0.0^{c}$ \\
RO $20-1724$ & 4.0 & $(n=2)$ \\
$\left(5 \times 10^{-5} \mathrm{M}\right)$ & $(n=1)$ & - \\
IBMX & 4.0 & \\
$\left(1 \times 10^{-4} \mathrm{M}\right)$ & $(n=1)$ & $0.3^{c}$ \\
Forskolin & 4.5 & $(n=1)$ \\
$\left(1 \times 10^{-6} \mathrm{M}\right)$ & $(n=2)$ &
\end{tabular}

"Treatments applied at CT 7-8.

" 1 hr treatments applied between CT 13 and CT 23 .

- Treatments applied at CT 16-17.

${ }^{d} p<0.001$.

A

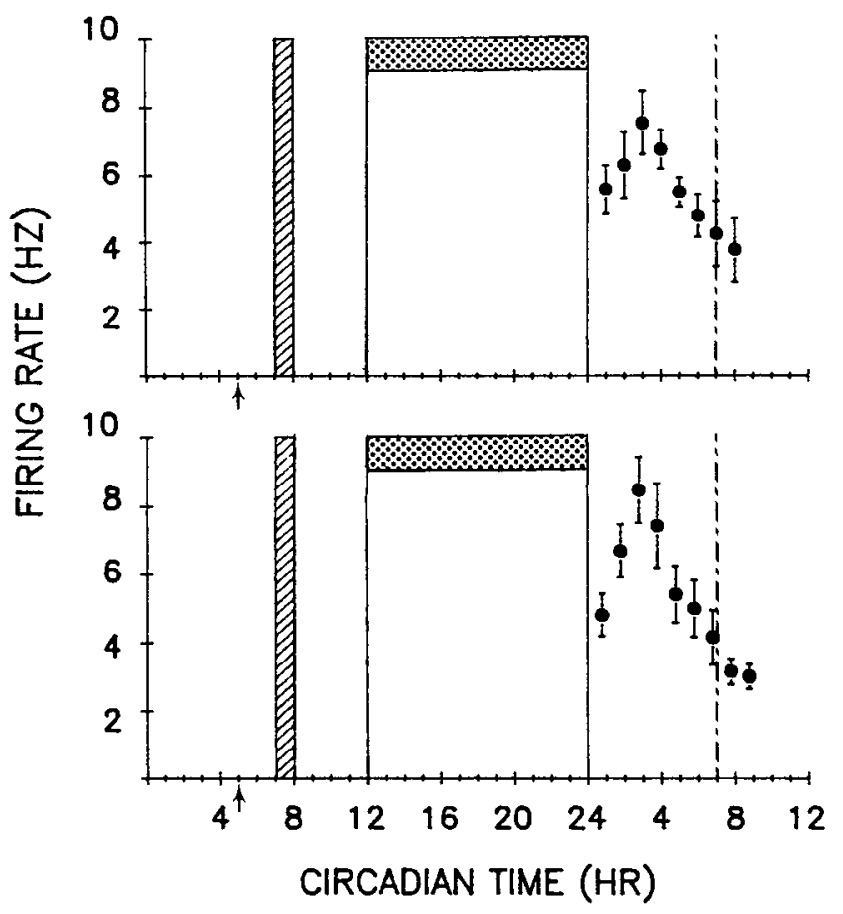

Figure 8. Treatments that increase endogenous cAMP levels mimic cAMP analogs. $A$, Slices were treated at CT 7-8 with $5 \times 10^{-5} \mathrm{M}$ RO 20-1724 on day 1 . Electrical activity on day 2 peaked at CT 3 , indicating a $4 \mathrm{hr}$ shift in the electrical activity rhythm. $B$, Slices were treated at CT 7-8 with $1 \times 10^{-6} \mathrm{M}$ forskolin on day 1 . Electrical activity on day 2 peaked at CT 2.75 , indicating a $4.25 \mathrm{hr}$ phase advance in the electrical activity rhythm. See Figure 1 and 2 legends for explanation of symbols.

tendrigh et al. (1958) showed by using double pulses of light that the Drosophila pacemaker is reset within $1 \mathrm{hr}$, even though it takes several days for the steady-state behavioral shift to manifest itself. The transient adjustments in behavioral rhythms seen in vivo after a phase-shifting stimulus may reflect slow processes involved in transforming a change in the pacemaker into shifts in the overt behavioral rhythms. A second possibility is that they reflect a disequilibrium between interacting oscillators, possibly due to feedback from brain regions that the SCN drive. In the present case, these forces are absent: we are measuring a direct expression of the oscillator in the apparent absence of feedback.

The phase shifts we see in vitro also differ in magnitude from those seen in vivo. Steady-state phase shifts of behavior in mammals after single stimuli rarely exceed $2 \mathrm{hr}$ (Daan and Pittendrigh, 1976), whereas in vitro we see phase shifts of 5 or $6 \mathrm{hr}$ in the pacemaker itself. This does not appear attributable to the phase-shifting stimulus used, since similar large phase shifts are seen after pulses of high $\mathrm{K}^{+}$in vitro (Gillette, 1987), as well as after preparing brain slices during lights-off (Gillette, 1986). Rather, it is again possible that the responsiveness of the SCN pacemaker to external stimuli is limited or dampened in the intact animal due to interactions with other brain regions but that these restrictions are removed when the SCN are isolated in a brain slice preparation. Thus, these results suggest a possible role of feedback circuits in normal SCN function.

Regarding the second contribution by these data to our understanding of the SCN pacemaker, several lines of evidence support the notion that the phase shifts seen after cAMP analog treatment are mediated by specific stimulation of cAMP path- 
ways. First, we found that 2 different analogs of cAMP produce similar phase advances of the electrical activity rhythm, whereas Br-5'-AMP does not affect the phase of the SCN pacemaker. Second, the dose-response curve indicates that an extremely small concentration of BA-cAMP is required to phase-shift the rhythm and that concentrations $10^{6}$ times larger produce essentially the same effect. Thus, BA-cAMP appears to be a very potent stimulus for resetting the pacemaker, but even at much higher levels it does not appear to produce side effects that interfere with its phase-shifting ability. Third, treatments that increase endogenous levels of cAMP phase-shift the SCN pacemaker to the same extent as active cAMP analogs, and they demonstrate a similar phase dependency in their ability to affect the pacemaker. Finally, the SCN pacemaker is sensitive to increases in cAMP stimulation only between CT 3 and 12 , while treatments at other times have no effect. Together these results lead us to conclude that the SCN pacemaker is specifically sensitive to daytime cAMP stimulation.

These findings have some correspondence with the effects of agents that stimulate cAMP pathways in the molluscan circadian pacemaker. In the snails Aplysia and Bulla, the clock is located in neurons at the base of the retina. These cells express a circadian rhythm of electrical activity for several days outside the animal (Jacklet, 1969; Block and Wallace, 1982). Like the mammalian SCN, the molluscan clock is sensitive to cAMP stimulation; however, the sensitivity appears to be much lower than in mammals. The clock rhythm of Aplysia is reset in vitro by $6 \mathrm{hr}$ pulses of cAMP analogs or by 5 -HT, which stimulates cAMP accumulation in the eye (Eskin et al., 1982). The maximal phase advance, induced by exposure at CT 7-13 to BA-cAMP $\left(2 \times 10^{-3} \mathrm{M}\right)$ is $3.25 \mathrm{hr}$ (compared with phase advances of up to $6 \mathrm{hr}$ for a $1 \mathrm{hr}$ exposure to cAMP analogs for rat SCN). Lesser advances are induced by agents affecting cAMP metabolism such as $6 \times 10^{-8}$ M RO 20-1724 (1.8 hr), $5 \times 10^{-7}$ M IBMX $(0.55 \mathrm{hr}), 2 \times 10^{-7} \mathrm{M}$ papaverine $(2.17 \mathrm{hr}$ : Eskin et al., 1982), or $1 \times 10^{-6} \mathrm{M}$ forskolin (2.0 hr: Eskin and Takahashi, 1983). Other types of molluscan neurons have been shown to be extremely sensitive to these agents (R. Gillette et al., 1982; Lotshaw and Levitan, 1987), suggesting that the differences in responsiveness are probably not due to a phylogenetic inability to respond.

Instead, they would appear to reflect differences in the organization of the molluscan and mammalian circadian systems. The molluscan clock seems to be less fine-tuned to changes in inputs since long exposures to high concentrations of the stimulus are needed to reset it. Furthermore, although the periods during which stimulation of cAMP-dependent pathways phaseadvance the pacemaker are very similar, molluscs show a second kind of response not seen in mammals. During the late subjective night and early morning (CT 22-4), $6 \mathrm{hr}$ exposure to BAcAMP induces phase delays of the neuronal activity rhythm averaging $3.75 \mathrm{hr}$. We have not explored the effects of treatments longer than $1 \mathrm{hr}$, but inasmuch as $5 \times 10^{-4} \mathrm{M}$ BA-cAMP for 1 $\mathrm{hr}$ is an extremely effective phase-advancing stimulus (Fig. 6) and we tested it throughout the circadian cycle (Fig. 4), as well as at $5 \times 10^{-8} \mathrm{M}$ at CT 1.5 , we should have encountered phase delays had they been inducible.

In the organism, environmental stimuli that reset the clock, such as changes in the lighting cycle, elicit both advances and delays depending upon the phase of the clock at which they are encountered. These stimuli are transmitted, both directly and indirectly, to the SCN where afferent axons make multiple syn- apses. Thus, unlike the situation in Aplysia, in which clock neurons respond directly to illumination (Eskin et al., 1982; Eskin and Takahashi, 1983), in mammals, there is synaptic processing between the photoreceptors and clock neurons. Internal circuitry deduced from anatomical studies of the SCN is complex (van den Pol and Gorcs, 1986). At least 21 neurotransmitters or neuromodulators have been immunocytochemically localized within the SCN or have been shown to affect SCN neuronal activity (Nishino and Koizumi, 1977; Groos et al., 1983; Moore, 1983; van den Pol and Tsujimoto, 1985; van den Pol and Gorcs, 1986); the role of none is yet understood. The potential for both afferent and internal information processing is therefore greater in the SCN than in the molluscan clock.

Moreover, evidence suggests that the SCN may process phase advances and delays differently. Peripheral injection of bicuculline, a selective GABA antagonist, into hamsters blocks the phase delays induced by light but does not alter light-induced phase advances (Kalph and Menaker, 1985). Conversely, peripheral injection of diazepam, a GABA antagonist with potent effect on second-messenger systems as well, blocks light-induecd phase advances but not phase delays (Ralph and Menaker, 1986). These results suggest that in mammals sensory inputs may be mediated differently, by separate pathways or receptors, at different times of the day. Our observation that cAMP induces only phase advances may relate to these findings: possibly stimulation of a specific subset of afferents, only between CT 3-12, generates increases in endogenous cAMP levels, which then lead to advancement of the clock. Thus, the differences in the clocks of mollusc and mammal, which may lie in the input pathways and/or in the internal circuitry of the pacemaker neuronal system, appear to make SCN responsiveness to cAMP simultaneously more sensitive and more temporally limited.

Several other treatments have been found to be day-active in their ability to phase-shift the circadian rhythms of mammals. These include dark pulses given to animals housed in constant light (Boulos and Rusak, 1982; Ellis et al., 1982) and injections of putative neurotransmitter neuropeptide Y (Albers and Ferris, 1984), the benzodiazepine Triazolam (Turek and Losee-Olson, 1986), and the protein synthesis inhibitor anisomycin (Inouye et al., 1985; Takahashi and Turek, 1987). In all 4 cases, however, the treatments produce both phase advances and phase delays and so do not entirely mimic the effects of cAMP analogs in vitro. Neverthelcss, it is possiblc that the mechanisms through which these treatments phase-advance the behavioral rhythms may include increases in the SCN's endogenous cAMP levels.

The phase-dependent sensitivity of the SCN pacemaker to increases in cAMP suggests that either cAMP acts within the pacemaker mechanism itself or else it acts within a pathway that has direct access to the pacemaker. One possibility is that cAMP normally acts within the SCN to change the phosphorylation state of proteins whose activity is part of the pacemaker mechanism. Support for this comes from evidence showing that the SCN exhibit day/night differences in protein synthesis and phosphorylation state (Gillette et al., 1986; Roberts and Moore, 1986). The importance of specific proteins to the SCN pacemaker is further supported by the ability of anisomycin to phaseshift circadian rhythms in vivo at phases similar to those when cAMP affects the pacemaker (Inouye et al., 1985; Takahashi and Turek, 1987).

Overall, these results are significant in that they emphasize the SCN's endogenous pacemaking properties. The findings that the pacemaker is stable over several cycles in glucose-supple- 
mented salt solution and can be reset in vitro underscore both the intrinsic nature of the phenomena we have observed and the potential for using this approach to elucidate mechanisms underlying the SCN clock. Further work should allow identification of cellular and molecular substrates associated with the phase-specific sensitivity of the suprachiasmatic pacemaker to cAMP.

\section{References}

Albers, H. E., and C. F. Ferris (1984) Neuropeptide Y: Role in light dark cycle entrainment of hamster circadian rhythms. Neurosci. Lett. 50: 163-168.

Block, G. D., and S. F. Wallace (1982) Localization of a circadian pacemaker in the eye of a mollusc, Bulla. Science 217: 155-157.

Boulos, Z., and B. Rusak (1982) Circadian phase response curves for dark pulses in the hamster. J. Comp. Physiol. 146: 411-417.

Chasin, M., and D. Harris (1976) Inhibitors and activators of cyclic nucleotide phosphodiesterase. Adv. Cyclic Nucleotide Res. 7: 225264.

Daan, S., and C. S. Pittendrigh (1976) A functional analysis of circadian pacemakers in nocturnal rodents. II. The variability of phase response curves. J. Comp. Physiol. 106: 253-266.

DeCoursey, P. J. (1964) Function of a light response rhythm in hamsters. J. Comp. Physiol. 63: 189-196.

Earnest, D. J., and C. D. Sladek (1986) Circadian rhythms of vasopressin release from individual rat suprachiasmatic explants in vitro. Brain Res. 382: 129-133.

Earnest, D. J., and C. D. Sladek (1987) Circadian vasopressin release from perifused rat suprachiasmatic explants in vitro: Effects of acute stimulation. Brain Res. 422: 398-402.

Ellis, G. B., R. E. McKlveen, and F. W. Turek (1982) Dark pulses affect the circadian rhythm of activity in hamsters kept in constant light. Am. J. Physiol. 242: R44-R50.

Eskin, A., and J. S. Takahashi (1983) Adenylate cyclase activation shifts the phase of a circadian pacemaker. Science 220:82-84.

Eskin, A., G. Corrent, C.-Y. Lin, and D. J. McAdoo (1982) Mechanisms for shifting the phase of a circadian rhythm by serotonin: Involvement of cAMP. Proc. Natl. Acad. Sci. USA 79: 660-664.

Gillette, M. (1985) Preparation of brain slices from the suprachiasmatic nuclei of rat can reset the circadian clock. J. Physiol. (Lond.) 369: 55P.

Gillette, M. U. (1986) The suprachiasmatic nuclei: Circadian shifts induced at the time of hypothalamic slice preparation are preserved in vitro. Brain Res. 379: 176-181.

Gillette, M. U. (1987) Effects of ionic manipulation on the circadian rhythm of neuronal firing rate in the suprachiasmatic brain slice. Soc. Neurosci. Abst. 13: 51 .

Gillette, M. U., and R. A. Prosser (1988) Circadian rhythm of the rat suprachiasmatic brain slice is rapidly reset by daytime application of cAMP analogs. Brain Res. 474: 348-352.

Gillette, M. U., and S. M. Reppert (1987) The hypothalamic suprachiasmatic nuclei: Circadian patterns of vasopressin secretion and neuronal activity in vitro. Brain Res. Bull. 19: 135-139.

Gillette, M. U., A. M. Reiman, and L. E. Lipeski (1986) Circadian protein and phosphoprotein changes in the suprachiasmatic nuclei: The difference between night and day. Soc. Neurosci. Abstr. 12: 845 .

Gillette, R., M. U. Gillette, and W. J. Davis (1982) Substrates of command ability in a buccal neuron of Pleurobranchaea. II. Potential role of cyclic AMP. J. Comp. Physiol. 146: 461-470.

Green, D. J., and R. Gillette (1982) Circadian rhythm of firing rate recorded from single cells in the rat suprachiasmatic slice. Brain Res. 245: $198-200$.

Groos, G., and J. Hendrics (1982) Circadian rhythms in electrical discharge of rat suprachiasmatic neurones recorded in vitro. Neurosci. Lett. 34: 283-288.

Groos, G., R. Mason, and J. Meyer (1983) Electrical and pharmacological properties of the suprachiasmatic nuclei. Fed. Proc. 42: 27902795.

Hatton, G. I., A. D. Doran, A. K. Salm, and C. D. Tweedle (1980) Brain slice preparation: Hypothalamus. Brain Res. Bull. 5: 405-414.

Hockberger, P., and Y. Yamane (1987) Compartmentalization of cyclic AMP elevation in neurons of Aplysia californica. Cell. Mol. Neurobiol. 7: 19-33.
Inouye, S.-I. T., and H. Kawamura (1979) Persistence of circadian rhythmicity in a mammalian hypothalamic "island" containing the suprachiasmatic nucleus. Proc. Natl. Acad. Sci. USA 76: 5962-5966.

Inouye, S. T., J. S. Takahashi, and F. W. Turek (1985) Anisomycin, injected either peripherally or directly into the suprachiasmatic nucleus (SCN), induces phase shifts in the circadian rhythm of locomotor activity in hamsters. Soc. Neurosci. Abstr. 11: 1139.

Jacklet, J. W. (1969) A circadian rhythm of optic nerve impulses recorded in darkness from the isolated eye of Aplysia. Science 164: $562-564$.

Liou, S. Y., S. Shibata, A. Shiratsuchi, and S. Ueki (1986) Effects of dibutyryl cyclic adenosine monophosphate and dibutyryl cyclic guanosine monophosphate on neuron activity of suprachiasmatic nucleus in rat hypothalamic slice preparation. Neurosci. Lett. 67: 339-343.

Lotshaw, D. P., and I. B. Levitan (1987) Serotonin and forskolin increase an inwardly rectifying potassium conductance in cultured identified Aplysia neurons. J. Neurophysiol. 58: 909-921.

Meyer, R. B., and J. P. Miller (1974) Analogs of cyclic AMP and cyclic GMP: General methods of synthesis and the relationship of structure to enzymatic activity. Life Sci. 14: 1019-1040.

Moore, R. Y. (1983) Organization and function of a central nervous system circadian oscillator: The suprachiasmatic hypothalamic nucleus. Fed. Proc. 42: 2783-2789.

Moore, R. Y., and V. B. Eichler (1972) Loss of a circadian adrcnal corticosterone rhythm following suprachiasmatic lesions in the rat. Brain Res. 42: 201-206.

Nishino, H., and K. Koizumi (1977) Responses of neurons in the suprachiasmatic nuclei of the hypothalamus to putative transmitters. Brain Res. 120: 167-172

Pittendrigh, C. S. (1960) Circadian rhythms and the circadian organization of living systems. Cold Spring Harbor Symp. Quant. Biol. $25 \cdot 159-184$

Pittendrigh, C. S., V. Bruce, and P. Kaus (1958) On the significance of transients in daily rhythms. Proc. Natl. Acad. Sci. USA 44:965973

Prosser, R. A., and M. U. Gillette (1986) cAMP analogs reset the circadian oscillator of rat SCN in vitro. Soc. Neurosci. Abstr. 12:211.

Ralph, M. R., and M. Mcnakcr (1985) Bicucullinc blocks circadian phase delays but not advances. Brain Res. 325: 362-365.

Ralph, M. R., and M. Menaker (1986) Effects of diazepam on circadian phase advances and delays. Brain Res. 372: 405-408.

Roberts, M. H., and R. Y. Moore (1986) Diurnal change of protein synthesis in the suprachiasmatic nucleus of the rat. Soc. Neurosci. Abstr. 12: 211.

Seamon, K. B., W. Padgett, and J. W. Daly (1981) Forskolin: Unique diterpene activator of adenylate cyclase in membranes and in intact cells. Proc. Natl. Acad. Sci. USA 78: 3363-3367.

Shibata, S., and R. Y. Moore (1988) Electrical and metabolic activity of suprachiasmatic neurons in hamster hypothalamic slices. Brain Res. 438: 374-378.

Shibata, S., Y. Oomura, H. Kita, and K. Hattori (1982) Circadian rhythmic changes in neuronal activity in the suprachiasmatic nucleus of the rat hypothalamic slice. Brain Res. 247: 154-158.

Steiner, A. L., R. E. Wehmann, C. W. Parker, and D. M. Kipnis (1972) Radioimmunoassay for measurement of cyclic nucleotides. Adv. $\mathrm{Cy}-$ clic Nucleotide Res. 2: 51-59.

Stephan, F. K., and I. Zucker (1972) Circadian rhythms in drinking behavior and locomotor activity of rats are eliminated by hypothalamic lesions. Proc. Natl. Acad. Sci. USA 69: 1583-1586.

Takahashi, J. S., and F. W. Turek (1987) Anisomycin, an inhibitor of protein synthesis, perturbs the phase of a mammalian circadian pacemaker. Brain Res. 405: 199-203.

Turek, F. W., and S. Losee-Olson (1986) A benzodiazepine used in the treatment of insomnia phase-shifts the mammalian circadian clock Nature 321: 167-168.

van den Pol, A. N., and T. Gorcs (1986) Synaptic relationships between neurons containing vasopressin, gastrin-releasing peptide, vasoactive intestinal peptide and glutamate decarboxylase immunoreactivity in the suprachiasmatic nucleus: Dual ultrastructural immunocytochemistry with gold-substituted silver peroxidase. J. Comp. Neurol. 252: 507-521.

van den Pol, A. N., and K. L. Tsujimoto (1985) Neurotransmitters of the hypothalamic suprachiasmatic nucleus: Immunocytochemical analysis of 25 neuronal antigens. Neuroscience 15: 1049-1086. 